\title{
Unusual Location of Coelomic Cysts
}

\author{
Smadhi Hanen 1,2,3*, Ben Jemai Emna ${ }^{1,2,3}$, Diouani Mohamed Fethi' ${ }^{4}$ Abdennadhar Mahdi' \\ Attia Monia6, Ayadi-Kaddour Aida7 , Megdiche Mohamed Lamine ${ }^{1,2,3}$
}

${ }^{1}$ Department of Pneumology Ibn Nafis, Abderrahman Mami University Hospital, Ariana, Tunisia

${ }^{2}$ Faculté de Médecine de Tunis, Université Tunis El Manar, Tunis, Tunisia

${ }^{3}$ Faculté des Sciences de Bizerte, Université de Carthage, Carthage, Tunisia

${ }^{4}$ Laboratoire d'Epidémiologie et de Microbiologie Vétérinaire Institut Pasteur de Tunis, Tunis, Tunisia

${ }^{5}$ Department of Thoracic Surgery, Abderrahman Mami University Hospital, Ariana, Tunisia

${ }^{6}$ Department of Radiology, Abderrahman Mami University Hospital, Ariana, Tunisia

${ }^{7}$ Department of Pathology, Abderrahman Mami University Hospital, Ariana, Tunisia

Email: *smadhi_hanen@yahoo.fr

How to cite this paper: Hanen, S., Emna, B.J., Fethi, D.M., Mahdi, A., Monia, A., Aida, A.-K. and Lamine, M.M. (2018) Unusual Location of Coelomic Cysts. Open Journal of Respiratory Diseases, 8, 33-37. https://doi.org/10.4236/ojrd.2018.83004

Received: July 2, 2018

Accepted: August 11, 2018

Published: August 14, 2018

Copyright (c) 2018 by authors and Scientific Research Publishing Inc. This work is licensed under the Creative Commons Attribution International License (CC BY 4.0).

http://creativecommons.org/licenses/by/4.0/

\begin{abstract}
Intrathoracic mesothelial cysts are congenital lesions due to an abnormal development of the pericardial coelom. They are generally unilocular and can develop in various sites. We describe a case of multicystic lesions in the anterior mediastinum of a 53-year-old woman discovered in a context of an encysted pleural effusion. Chest computed tomography (CT) scan revealed multiple cystic lesions in the anterior and posterior mediastinum. Surgery was carried out and histopathology and immunohistochemistry confirmed the diagnosis of multiple coelomic cysts of the mediastinum.
\end{abstract}

\section{Keywords}

Mesothelial, Cysts, Mediastinum, Surgery, Pathology

\section{Introduction}

Primary cysts of the mediastinum regroup bronchogenic cysts, enteric or duplication cysts, mesothelial cysts, thymic cysts, and thoracic duct cysts [1]. Coelomic cysts are rare with mesothelial origin. They are often discovered incidentally but sometimes with indistinct symptoms in varying parts of the body. They are generally unilocular and can increase in various sites [2]. Mediastinal multicystic mesothelial cysts have been rarely reported.

\section{Case Report}

We report a case of a 53-year-old woman, with no significant past medical history, no smoker, was referred to our department with a history of chest pain, cough and 
purulent expectoration during a period of 2 months. Physical examination found a right pleuritic syndrome. The biological examinations were free of anomalies. Chest X-ray showed a paracardiac right basal opacity. Thoracic ultrasound showed a left anterior mediastinal multicystic mass, associated with pleural effusion encysted on the right. Chest computed tomography (CT) scan revealed mediastinal fluid formations, bilateral anterior and posterior extending in retro-cardiac to the right hemithorax in the anterior and posterior extrapleural space (Figure 1). The lesions were not enhanced by intravenous contrast. However, the rest of the lung fields appeared clear. Magnetic resonance imaging (MRI) (Figure 2) showed anterior and posterior mediastinal multicystic formations seen on the CT scan. The cyst was surgically removed through a posterolateral thoracotomy which revealed several cystic formations of variable size with a serohematic content occupying all the mediastinal lodges (Figure 3). The cysts had no contact with the pericardium or the right lobe of the thymus. They appeared to have a very thin wall through which approximately $500 \mathrm{~mL}$ of straw-colored fluid was aspirated and sent for analysis. Histopathological examination confirmed that there were mesothelial cysts (Figure 4). The patient had an uneventful postoperative course and was discharged on postoperative day 10. Surgical resection of the cysts leads to relief of the thoracic pain over a one-year follow-up period.

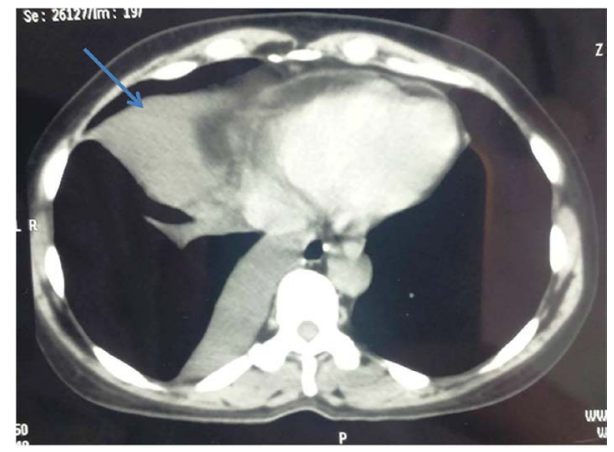

Figure 1. Chest computed tomography (CT) scan revealed mediastinal fluid formations, bilateral anterior and posterior extending in retro-cardiac to the right hemithorax in the anterior and posterior extrapleural space.

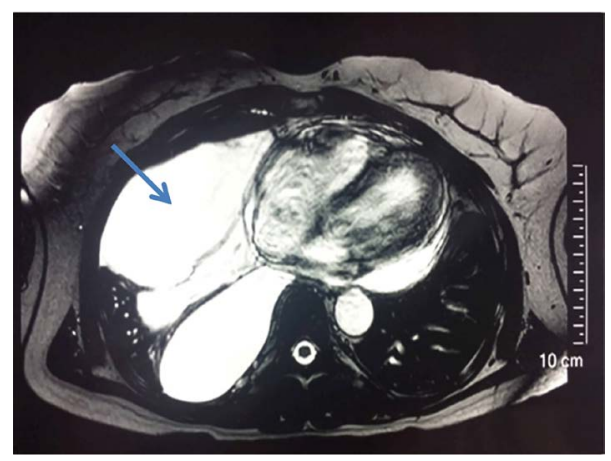

Figure 2. Magnetic resonance imaging of the mediastinal showed mediastinal multicystic formations in the right hemithorax extending to the anterior and posterior axtrapleural espace. 


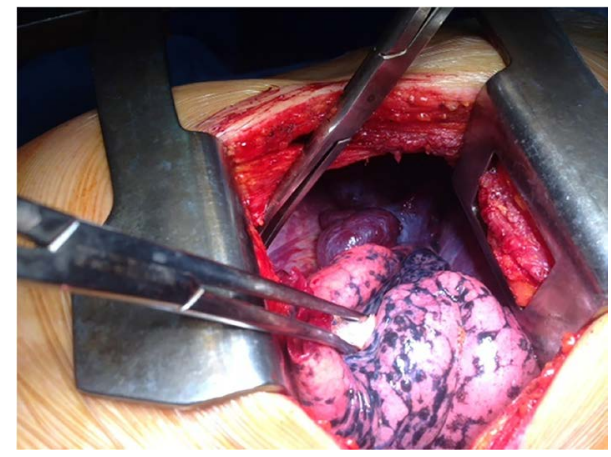

Figure 3. Posterolateral thoracotomy revealed several cystic formations of variable size with a serohematic content occupying all the medistinal lodges.

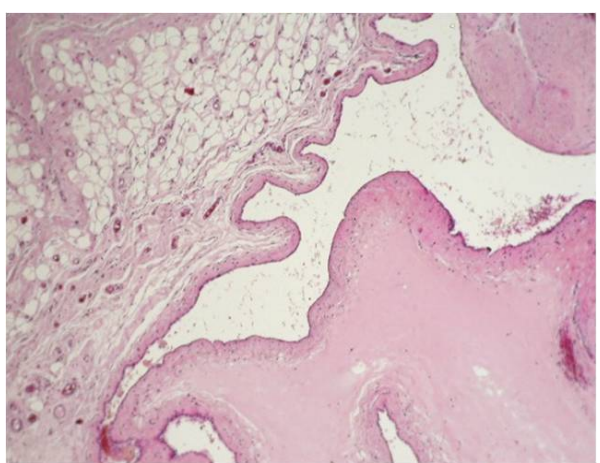

Figure 4. Histology: Adipose tissue surmounted by a rather thin fibrous wall whose inner border is lined by a regular simple flattened and cubic epithelium, without cytonuclear atypies (HEX100).

\section{Discussion}

Mesothelial cysts are benign lesions typically lined by a single layer of mesothelial cells [3]. These congenital or acquired cysts may be found adjacent to serous membranes all over the body but they are rarely encountered in the thorax [4]. These cysts are usually comprised of a benign fluid filled sac, lined with flattened or cuboidal epithelium. Mesothelial cysts were originally thought to be the result of an anomalous development of the ventral recessus of the parietal primitive pericardial cavity. According to this theory, the cyst is usually uniloculated. Nevertheless, Cangemi, et al. reported in their study, that 23 of 24 pericardial cysts were unilocular and only 1 was multilocular [5]. They are classically situated in the anterior mediastinum, in the right cardiophrenic angle in 51\%-70\% of cases, and in $22 \%$ - $38 \%$ of cases they are located in the left cardiophrenic angle [6]. Coelomic cysts represent $4 \%$ to $11 \%$ of mediastinal tumors and $22 \%$ to $38 \%$ of mediastinal cysts. They result from a lack of fusion of the primary coelomic mesenchymal gaps. Pleuropericardial cysts are the most frequent coelomic cysts [7]. The clinical presentation is not specific. They are usually discovered incidentally on imaging exams or with otherwise vague symptoms in varying parts of the body. Clinical signs can be observed in a little more than a third of the cases especially in the presence of very large cysts responsible for 
compression phenomena on the neighborhood organs. Our patient presented a history of chest pain, cough and purulent expectoration associated with a right pleuritic syndrome at physical examination. Several differential diagnoses can be evoked face to this cystic lesion of the mediastinum. In our patient, we first evoked a mediastinal hydatid cyst given the endemicity of this pathology in our country. In addition, other diagnoses were possible such as a bronchogenic cyst, a cystic lymphangioma or a thymic cyst. Radiological exams do not formally make the diagnosis, so the histological confirmation of the cyst is necessary. Surgical resection should be reserved for symptomatic or atypical cysts. Complex cysts such as those in abnormal locations with multiple loculations, high attenuation within the cyst, or a mass-like component to the cyst, require resection for diagnosis. Ordinarily, this can be performed simply through a limited thoracotomy or thoracoscopic approach, with least morbidity and low mortality. These last years, progress in video-assisted thoracoscopic surgery (VATS) has enabled us to use this technique for simultaneous diagnosis and treatment [8]. In our case, the localization of the cysts had justified a thoracotomy. Resection is curative in virtually all cases.

\section{Conclusion}

Coelomic cysts are very rare malformations of embryonic origin of the mediastinum. Ectopic localization in both posterior and anterior mediastinum associated with a right encysted pleural effusion is exceptional.

\section{Funding}

This research received no specific grant from any funding agency in the public, commercial, or not-for-profit sectors.

\section{Conflicts of Interest}

The authors declare no conflicts of interest regarding the publication of this paper.

\section{References}

[1] Sasaki, H., Yano, M., Kiriyama, M., Kaji, M., Fukai, I., Yamakawa, Y., et al. (2003) Multicystic Mesothelial Cyst of Mediastinum. Surgery Today, 33, 199-201. https://doi.org/10.1007/s005950300044

[2] Augustine, A.J., Pai, R.K., Pai, R., Diaz, E.A., Prabhu, H.S. and Swaroop, R. (2001) Mesothelial Cyst at Porta Hepatis. Indian Journal of Gastroenterology, 20, 35-36.

[3] Shields, T.W. (2001) Mesothelial and Other Less Common Cysts of the Mediastinum. In: Shields, T.W., Lo Cicero III, J. and Ponn, R.B., Eds., General Thoracic Surgery, Lippincott Williams and Wilkins, Philadelphia, PA.

[4] Smith, V.C., Edwards, R.A., Jorgensen, J.L., Goldfarb, R.A., Kadmon, D., Cagle, P., et al. (2000) Unilocular Retroperitoneal Cyst of Mesothelial Origin Presenting as a Renal Mass. Archives of Pathology \& Laboratory Medicine, 124, 766-769.

[5] Cangemi, V., Volpino, P., Gualdi, G., Polettini, E., Frati, R., Cangemi, B., et al. 
(1999) Pericardial Cysts of the Mediastinum. The Journal of Cardiovascular Surgery, 40, 909-913.

[6] Feigin, D.S., Fenoglio, J.J., McAllister, H.A. and Madewell, J.E. (1977) Pericardial Cysts: A Radiologic-Pathologic Review. Radiology, 125, 15-20.

https://doi.org/10.1148/125.1.15

[7] Le Pimpec-Barthes, A., Cazes, P., Bagan, A., Badia, C., Vlas, A., Hernigou, et al. (2010) Les kystes du médiastin: Approche diagnostique et traitement. Revue de Pneumologie Clinique, 66, 52-62. https://doi.org/10.1016/j.pneumo.2009.12.009

[8] Roviaro, G., Rebuffat, C., Varoli, F., Vergani, C., Maciocco, M. and Scalambra, S. (1994) Videothoracoscopic Excision of Cysts of Mediastinal Masses: Indications and Technique. The Annals of Thoracic Surgery, 58, 1679-1683.

https://doi.org/10.1016/0003-4975(94)91658-6 